\title{
A Class of Quantum LDPC Codes Constructed From Finite Geometries
}

\author{
Salah A. Aly \\ Department of Computer Science, Texas A\&M University \\ College Station, TX 77843, USA \\ Email: salah@cs.tamu.edu
}

\begin{abstract}
Low-density parity check (LDPC) codes are a significant class of classical codes with many applications. Several good LDPC codes have been constructed using random, algebraic, and finite geometries approaches, with containing cycles of length at least six in their Tanner graphs. However, it is impossible to design a self-orthogonal parity check matrix of an LDPC code without introducing cycles of length four.

In this paper, a new class of quantum LDPC codes based on lines and points of finite geometries is constructed. The parity check matrices of these codes are adapted to be selforthogonal with containing only one cycle of length four in each pair of two rows. Also, the column and row weights, and bounds on the minimum distance of these codes are given. As a consequence, these codes can be encoded using shift-register encoding algorithms and can be decoded using iterative decoding algorithms over various quantum depolarizing channels.
\end{abstract}

\section{INTRODUCTION}

Low density parity check (LDPC) codes are a capacityapproaching (Shannon limit) class of codes that were first described in a seminal work by Gallager [8]. In Tanner [21], LDPC codes were rediscovered and presented in a graphical interpretation (codes over graphs). Iterative decoding of LDPC and turbo codes highlighted the importance of these classes of codes for communication and storage channels. Furthermore, they have been used extensively in many applications [7], [15], [16].

There have been several notable attempts to construct regular and irregular good LDPC codes using algebraic combinatorics and random constructions, see [16], [20], and references therein. Liva et al. [16] presented a survey of the previous work done on algebraic constructions of LDPC codes based on finite geometries, elements of finite fields, and RS codes. Furthermore, a good construction of LDPC codes should have a girth of the Tanner graph, of at least six [15], [16].

Quantum information is sensitive to noise and needs error correction, control, and recovery strategies. Quantum block and convolutional codes are means to protect quantum information against noise and decoherence. A well-known class of quantum codes is called stabilize codes, in which it can be easily constructed using self-orthogonal (or dual-containing) classical codes, see [3], [5], [11] and references therein. Recently, subsystem codes combine the features of decoherence free subspaces, noiseless subsystems, and quantum errorcorrecting codes, see [2], [4], [13], [14] and references therein.

Quantum block LDPC codes have been proposed in [17], [18]. MacKay et al. in [17] constructed sparse graph quantum
LDPC codes based on cyclic matrices and using a computer search. Recently, Camera el al. derived quantum LDPC codes in an analytical method [6]. Hagiwara and Imai constructed quasi-cyclic (QC) LDPC codes and derived a family of quantum QC LDPC codes from a nested pair of classical codes [10].

In this paper, we construct LDPC codes based on finite geometry. We show that the constructed LDPC codes have quasi-cyclic structure and their parity check matrices can be adapted to satisfy the self-orthogonal (or dual-containing) conditions. The motivations for this work are that (i) LDPC codes constructed from finite geometries can be encoded using linear shift-registers. The column weights remain fixed with the increase in number of rows and length of the code. (ii) The adapted parity check matrix has exactly one cycle with length four between any two rows and many cycles with length of at least six. (iii) A class of quantum LDPC codes is constructed that can be decoded using known iterative decoding algorithms over quantum depolarizing channels; some of these algorithms are stated in [19].

Notation: Let $q$ be a prime power $p$ and $\mathbf{F}_{q}$ be a finite field with $q$ elements. Any two binary vectors $\mathbf{v}=\left(v_{1}, v_{2}, \ldots, v_{n}\right)$ and $\mathbf{u}=\left(u_{1}, u_{2}, \ldots, u_{n}\right)$ are orthogonal if their inner product vanishes, i.e., $\sum_{i=1}^{n} v_{i} u_{i} \bmod 2=0$. Let $\mathbf{H}$ be a parity check matrix defined over $\mathbf{F}_{2}$, then $\mathbf{H}$ is self-orthogonal if the inner product between any two arbitrary rows of $\mathbf{H}$ vanishes.

\section{LDPC CODE CONSTRUCTIONS AND Finite GEOMETRIES}

\section{A. LDPC Codes}

Definition 1: An $(\rho, \lambda)$ regular LDPC code is defined by a sparse binary parity check matrix $\mathbf{H}$ satisfying the following properties.

i) $\rho$ is the number of one's in a column.

ii) $\lambda$ is the number of one's in a row.

iii) Any two rows have at most one nonzero element in common. The code does not have cycles of length four in its Tanner graph.

iv) $\rho$ and $\lambda$ are small in comparison to the number of rows and length of the code. In addition, rows of the matrix $\mathbf{H}$ are not necessarily linearly independent.

The third condition guarantees that iterative decoding algorithms such as sum-product or message passing perform well over communication channels. In general it is hard to design 
regular LDPC satisfying the above conditions, see [15], [16], [20] and references therein.

\section{B. Finite Geometry}

Finite geometries can be classified into Euclidean and projective geometry over finite fields. Finite geometries codes are an important class of cyclic and quasi-cyclic codes because their encoder algorithms can be implemented using linear feedback shift registers and their decoder algorithms can be implemented using various decoding algorithms such as majority logic (MLG), sum-product (SPA), and weighted BF, see [12], [15], [16].

Definition 2: A finite geometry with a set of $n$ points $\left\{p_{1}, p_{2}, \ldots, p_{n}\right\}$, a set of $l$ lines $\left\{L_{1}, L_{2}, \ldots, L_{l}\right\}$ and an integer pair $(\lambda, \rho)$ is defined as follows:

i) Every line $L_{i}$ passes through $\rho$ points.

ii) Every point $p_{i}$ lies in $\lambda$ lines, i.e., every point $p_{i}$ is intersected by $\lambda$ lines.

iii) Any two points $p_{1}$ and $p_{j}$ can define one and only one line $L_{k}$ in between.

iv) Any two lines $L_{i}$ and $L_{j}$ either intersect at only one point $p_{i}$ or they are parallel.

Therefore, we can form a binary matrix $\mathbf{H}=\left[h_{i, j}\right]$ of size $l \times n$ over $\mathbf{F}_{2}$. The rows and columns of $\mathbf{H}$ correspond the $l$ lines and $n$ points in the Euclidean geometry, respectively. If the ith line $L_{i}$ passes through the point $p_{i}$ then $h_{i, j}=1$, and otherwise $h_{i, j}=0$ Fig. 1 shows an example of Euclidean

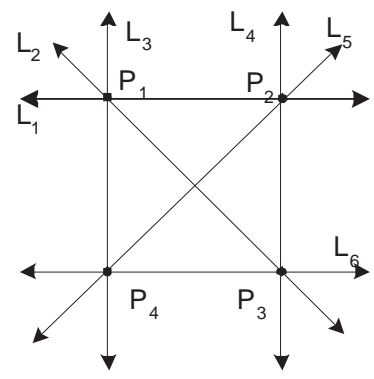

Fig. 1. Euclidean geometry with points $n=4$ and lines $l=6$

geometry with $n=4, l=6, \lambda=3$, and $\rho=2$. We can construct the incidence matrix $\mathbf{H}$ based on this geometry where every point and line correspond to a column and row, respectively. For $\rho<<l$ and $\lambda<<n$, The matrix $\mathbf{H}$ is a sparse low density parity check matrix. In this example, the matrix $\mathbf{H}_{E G-I}$ is given by

$$
\mathbf{H}_{E G-I}=\left(\begin{array}{cccc}
1 & 1 & 0 & 0 \\
1 & 0 & 1 & 0 \\
1 & 0 & 0 & 1 \\
0 & 1 & 1 & 0 \\
0 & 1 & 0 & 1 \\
0 & 0 & 1 & 1
\end{array}\right)
$$

We call the Euclidean geometry defined in this type as a Type-I EG. The Tanner graph of Type-I EG is a regular bipartite graph with $n$ code variable vertices and $l$ check-sum

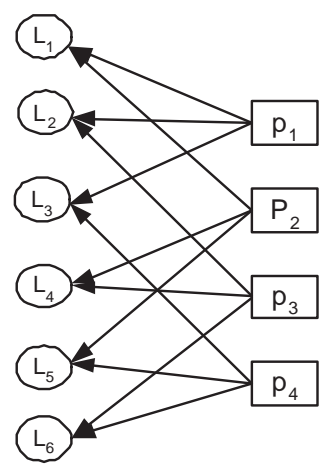

(a)

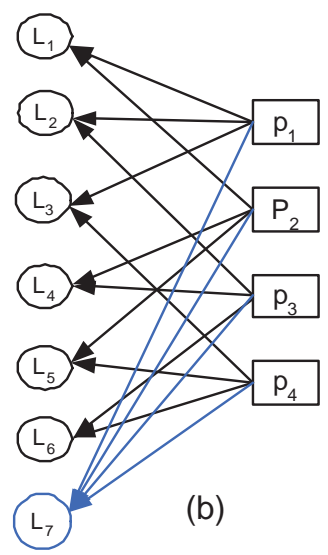

Fig. 2. (a) Euclidean geometry with $n=4$ points (check-sum) and $l=6$ lines (code variables) (b) This Tanner graph corresponds to a self-orthogonal parity check matrix.

vertices. Also, each variable bit vertex has degree $\lambda$ and each check-sum has degree $\rho$.

If we can take the transpose of this matrix $\mathbf{H}_{E G-I}$, then we can also define a $(\rho, \lambda)$ LDPC code with length $l$ and minimum distance is at least $\rho+1$. The codes defined in this type are called LDPC codes based on Type-II EG. In this type, any two rows intersect at exactly one position.

\section{Adapting the Matrix $\boldsymbol{H}_{E G-I I}$ to be Self-orthogonal}

Let $\mathbf{H}_{E G-I I}$ be a parity check matrix of a regular LDPC code constructed based on Type-II EG Euclidean geometry. We can construct a self-orthogonal matrix $\mathbf{H}_{E G-I I}^{\text {orth }}$ from $\mathbf{H}_{E G-I I}$ in two cases.

Case 1. If the number of one's in a row is odd and any two rows intersect at exactly one position, i.e., any line connects two points. As shown in Fig. 2, the Tanner graph corresponds to a self-orthogonal parity check matrix $\mathbf{H}_{E G-I I}^{\text {orth }}$ if and only if every check-sum has even degree and any any two checksum nodes meet at even code variable nodes. This condition is the same as every row in the parity check matrix $\mathbf{H}_{E G-I I}^{\text {orth }}$ has an even weight and any two rows overlap in even nonzero positions.

$$
\mathbf{H}_{E G-I I}^{\text {orth }}=\left(\mathbf{H}^{T} \mid \mathbf{1}\right)
$$

The vector $\mathbf{1}$ of length $n$ is added as the last column in $\mathbf{H}_{E G-I I}^{\text {orth }}$.

Case 2. Assume the number of one's in a line is even and any two rows intersect at exactly one position. We can construct a self-orthogonal parity check matrix $\mathbf{H}_{E G-I I}^{\text {orth }}$ as follows. We add the vector $\mathbf{1}$ along with the identity matrix $\mathbf{I}$ of size $n \times n$. We guarantee that any two rows of the matrix $\mathbf{H}_{E G-I I}^{\text {orth }}$ intersect at two nonzero positions and every row has an even weight.

$$
\mathbf{H}_{E G-I I}^{\text {orth }}=\left(\mathbf{H}^{T}|\mathbf{1}| \mathbf{I}\right) .
$$




\section{Characteristic Vectors and Matrices}

Let $n$ be a positive integer such that $n=q^{m}-1$, where $m=\operatorname{ord}_{n}(q)$ is the multiplicative order of $q$ modulo $n$. Let $\alpha$ denote a fixed primitive element of $\mathbf{F}_{q^{m}}$. Define a map $\mathbf{z}$ from $\mathbf{F}_{q^{m}}^{*}$ to $\mathbf{F}_{2}^{n}$ such that all entries of $\mathbf{z}\left(\alpha^{i}\right)$ are equal to 0 except at position $i$, where it is equal to 1 . For example, $\mathbf{z}\left(\alpha^{2}\right)=$ $(0,1,0, \ldots, 0)$. We call $\mathbf{z}\left(\alpha^{k}\right)$ the location (or characteristic) vector of $\alpha^{k}$. We can define the location vector $\mathbf{z}\left(\alpha^{i+j+1}\right)$ as the right cyclic shift of the location vector $\mathbf{z}\left(\alpha^{i+j}\right)$, for $0 \leq j \leq n-1$, and the power is taken module $n$. The location vector can be extended to two or more nonzero positions. for example, the location vector of $\alpha^{2}, \alpha^{3}$ and $\alpha^{5}$ is given by $\mathbf{z}\left(\alpha^{2}, \alpha^{3}, \alpha^{5}\right)=(0,1,1,0,1,0, \ldots, 0)$.

Definition 3: We can define a map $A$ that associates to an element $\mathbf{F}_{q^{m}}^{*}$ a circulant matrix in $\mathbf{F}_{2}^{n \times n}$ by

$$
A\left(\alpha^{i}\right)=\left(\begin{array}{c}
\mathbf{z}\left(\alpha^{i}\right) \\
\mathbf{z}\left(\alpha^{i+1}\right) \\
\vdots \\
\mathbf{z}\left(\alpha^{i+n-1}\right)
\end{array}\right) .
$$

By construction, $A\left(\alpha^{k}\right)$ contains a 1 in every row and column.

We will use the map $A$ to associate to a parity check matrix $H=\left(h_{i j}\right)$ in $\left(\mathbf{F}_{q^{m}}^{*}\right)$ the (larger and binary) parity check matrix $\mathbf{H}=\left(A\left(h_{i j}\right)\right)$ in $\mathbf{F}_{2}^{n \times n}$. The matrices $A\left(h_{i j}\right)^{\prime} s$ are $n \times n$ circulant permutation matrices based on some primitive elements $h_{i j}$ as shown in Definition 3.

\section{Constructing Self-Orthogonal Cyclic LDPC CODES FROM EUCLIDEAN GEOMETRY}

In this section we construct self-orthogonal algebraic Low Density Parity Check (LDPC) codes based on finite geometries. Particulary, there are two important classes of finite geometries: Euclidean and projective geometry.

\section{A. Euclidean Geometry $E G(m, q)$}

We construct regular LDPC codes based on lines and points of Euclidean geometry. The class we derive has a cyclic structure, so it is called cyclic LDPC codes. Cyclic LDPC codes can be defined by a sparse parity check matrix or by a generator polynomial and can be encoded using shift-register. Furthermore, they can be decoded using well-known iterative decoding algorithms [15], [16].

Let $q$ be power of a prime $p$, i.e. $q=p^{s}$ for some integer $s \geq 2$. Let $E G(m, q)$ be the m-dimensional Euclidean geometry over $\mathbf{F}_{q}$ for some integer $m \geq 2$. It consists of $p^{m s}=q^{m}$ points and every point is represented by an $\mathrm{m}$ tuple, see [12]. A line in $E G(m, q)$ can be described by a 1 -dimensional subspace of the vector space of all $m$-tuples over $\mathbf{F}_{q}$ or a coset of it. The number of lines in $E G(m, q)$ is given by

$$
\left(q^{m-1}\right)\left(q^{m}-1\right) /(q-1)
$$

and each line passes through $q$ points. Every line has $q^{(m-1)}-$ 1 lines parallel to it. Also, for any point in $E G(m, q)$, there are

$$
\left(q^{m}-1\right) /(q-1)
$$

lines intersect at this point. Two lines can intersect at only one point or they are parallel.

Let $\mathbf{F}_{q^{m}}$ be the extension field of $\mathbf{F}_{q}$. We can represent each element in $\mathbf{F}_{q^{m}}$ as an $m$-tuple over $\mathbf{F}_{q}$. Every element in the finite field $\mathbf{F}_{q^{m}}$ can be looked as a point in the Euclidean geometry $E G(m, q)$, henceforth $\mathbf{F}_{q^{m}}$ can be regarded as the Euclidean geometry $E G(m, q)$.

Let $\alpha$ be a primitive element of $\mathbf{F}_{q^{m}} \cdot q^{m}$ points of $E G(m, q)$ can be represented by elements of the set $\left\{0,1, \alpha, \alpha^{2}, \ldots, \alpha^{q^{m}-2}\right\}$. We can also define a line $L$ as the set of points of the form $\left\{\mathbf{a}+\gamma \mathbf{b} \mid \gamma \in \mathbf{F}_{q}\right\}$, where $\mathbf{a}$ and $\mathbf{b}$ are linearly independent over $\mathbf{F}_{q}$. For a given point $\mathbf{a}$, there are $\left(q^{m}-1\right) /(q-1)$ lines in $E G(m, q)$ that intersect at a.

Type-I EG. Let $n=q^{m}-1$ be the number of points excluding the original point $\mathbf{0}$ in $E G(m, q)$. Assume $L$ be a line not passing through $\mathbf{0}$. We can define the binary vector

$$
\mathbf{v}_{L}=\left(v_{1}, v_{1}, \ldots, v_{n}\right),
$$

where $v_{i}=1$ if the point $\alpha^{i}$ lies in a line $L$. The vector $\mathbf{v}_{L}$ is called the incidence vector of $L$. Elements of the vector $\mathbf{v}_{L}$ correspond to the elements $1, \alpha, \alpha^{2}, \ldots, \alpha^{n-1} . \alpha L$ is also a line in $E G(m, q)$, therefore $\alpha \mathbf{v}_{L}$ is a right cyclic-shift of the vector $\mathbf{v}_{L}$. Clearly, the lines $L, \alpha L, \ldots, \alpha^{n-1} L$ are all different. But, they may not be linearly independent.

Consider the vectors $L_{i}, \alpha L_{i}, \ldots, \alpha^{n-1} L_{i}$. We can construct an $n \times n$ matrix $H_{i}$ in the form

$$
H_{i}=\left(\begin{array}{c}
\mathbf{v}_{L_{i}} \\
\alpha \mathbf{v}_{L_{i}} \\
\vdots \\
\alpha^{n-1} \mathbf{v}_{L_{i}}
\end{array}\right)
$$

Clearly, $H_{i}$ is a circulant matrix with column and row weights equals to $q$, the number of points that lie in a line $\alpha^{j} L_{i}$, for $0 \leq j \leq n-1 . H_{i}$ has size of $n \times n$. The total number of lines in $E G(m, q)$ that do not pass through the origin $\mathbf{0}$ are given by

$$
\left(q^{m-1}-1\right)\left(q^{m}-1\right) /(q-1)
$$

They can be partitioned into $\left(q^{m-1}-1\right) /(q-1)$ cyclic classes, see [16]. Every class $\mathcal{H}_{i}$ can be defined by an incidence vector $L_{i}$ as $\left\{L_{i}, \alpha L_{i}, \alpha^{2} L_{i}, \ldots, \alpha^{n-1} L_{i}\right\}$ for $1 \leq i \leq\left(q^{m-1}-\right.$ $1) /(q-1)$. Let $1 \leq \ell \leq\left(q^{m-1}-1\right) /(q-1)$, then $\mathcal{H}_{E G, \ell}$ is defined as

$$
\mathcal{H}_{E G, \ell}=\left[\begin{array}{llll}
\mathcal{H}_{1} & \mathcal{H}_{2} & \ldots & \mathcal{H}_{\ell}
\end{array}\right]^{T} .
$$

For each cyclic class $\mathcal{H}_{i}$, we can form the matrix $\mathbf{H}_{i}$ over $\mathbf{F}_{2}$ of size $n \times n$. Therefore, $\mathbf{H}_{i}$ is a circulant binary matrix of row and column weights of $\mathrm{q}$.

If we assume that there are $1 \leq \ell \leq\left(q^{m-1}-1\right) /(q-1)$ incidence lines in $E G(m, q)$ not passing through the origin, then we can form the binary matrix 


$$
\mathbf{H}_{E G, \ell}=\left[\begin{array}{llll}
\mathbf{H}_{1} & \mathbf{H}_{2} & \ldots & \mathbf{H}_{\ell}
\end{array}\right]^{T} .
$$

The matrix $\mathbf{H}_{E G, \ell}$ consists of a $\ell$ sub-matrices $\mathbf{H}_{i}$ of size $n \times n$ and it has column and row weights $\ell q$ and $q$, respectively. The null space of the matrix $\mathbf{H}_{E G, \ell}$ gives a cyclic EG-LDPC code of length $n=q^{m}-1$ and minimum distance $\ell q+1$, whose Tanner graph has a girth of at least six, see [16], [20].

The Tanner graph of Type-I EG is a regular bipartite graph with $q^{m}-1$ code variable vertices and $l$ check-sum vertices. Also, Each variable bit vertex has degree $\rho=q$ and each check-sum has degree $\lambda=\ell q$.

Type-II EG. We can take the transpose of the parity check matrix $\mathcal{H}_{(E G, \ell)}$ over $\mathbf{F}_{q^{m}}$ as defined in Type-I to define a new parity check matrix with the following properties, see [12].

$$
\mathcal{H}_{E G, \ell}^{T}=\left[\begin{array}{llll}
\mathcal{H}_{1}^{T} & \mathcal{H}_{2}^{T} & \ldots & \mathcal{H}_{\ell}^{T}
\end{array}\right]
$$

So, the matrix $\mathcal{H}_{i}^{T}$ is the transpose matrix of $\mathcal{H}_{i}$. Consequently, we can define the binary matrix $\mathbf{H}_{E G, \ell}$

$$
\mathbf{H}_{E G, \ell}^{T}=\left[\begin{array}{llll}
\mathbf{H}_{1}^{T} & \mathbf{H}_{2}^{T} & \ldots & \mathbf{H}_{\ell}^{T}
\end{array}\right] .
$$

Let $\ell=\left(q^{m-1}-1\right) /(q-1)$, then the matrix $\mathbf{H}_{E G, \ell}^{T}$ has the following properties

i) The total number of columns is given by $\ell n=\left(q^{m-1}-\right.$ 1) $\left(q^{m}-1\right) /(q-1)$.

ii) Number of rows is given by $n=q^{m}-1$.

iii) The rows of this matrix correspond to the nonorigin points of $E G(m, q)$ and the columns correspond to the lines in $E G(m, q)$ that do not pass through the origin.

iv) $\lambda=\ell q=q\left(q^{m-1}-1\right) /(q-1)=\left(q^{m}-1\right) /(q-1)-1$ is the row weight for $\ell=\left(q^{m-1}-1\right) /(q-1)$. Also $\rho=q$ is the column weight.

v) Any two rows in $\mathbf{H}_{E G, \ell}^{T}$ have exactly one nonzero element in common. Also, any two columns have at most one nonzero element in common.

vi) The binary sub-matrix $\mathbf{H}_{i}^{T}$ has size $\left(q^{m}-1\right) \times\left(q^{m}-1\right)$. Also, it can be constructed using only one vector $\mathbf{v}_{L}$ that will be cyclically shifted $q^{m}-1$ times.

\section{B. QC LDPC Codes}

The matrix $\mathbf{H}_{E G, \ell}^{T}$ defines a quasi-cyclic (QC) LDPC code of length $N=\ell n=\left(q^{(m-1)}-1\right)\left(q^{m}-1\right) /(q-1)$ for $\ell=$ $\left(q^{m-1}-1\right) /(q-1)$. The matrix $\mathbf{H}_{E G, \ell}^{T}$ has $n=q^{m}-1$ rows that are not necessarily independent. We can define a QC LDPC code over $\mathbf{F}_{2}$ as the null-space of the matrix $\mathbf{H}_{E G, \ell}^{T}$ of sparse circulant sub-matrices of equal size. The matrix $\mathbf{H}_{E G, \ell}^{T}$ with parameters $(\rho, \lambda)$ has the following properties.

i) $\rho=q$ is the weight of a column $c_{i}$. $\rho$ does not depend on $m$, hence length of the code can be increased without increasing the column weight.

ii) $\lambda=\ell q$ is the weight of a row $r_{i}$. $\lambda$ depends on $m$, but the length of the code increases much faster than $\lambda$. iii) Every two columns intersect at most at one nonzero position. Every two rows have exactly one and only one nonzero position in common.

From this definition, the minimum distance of the LDPC code defined by the null-space of $\mathbf{H}_{E G, \ell}^{T}$ is at least $\rho+1$. This is because we can add at least $\rho+1$ columns in the parity check matrix $\mathbf{H}_{E G, \ell}^{T}$ to obtain the zero column (rank of $\mathbf{H}_{E G, \ell}^{T}$ is at least $(\rho+1))$. Furthermore, the girth of the Tanner graph for this matrix $\mathbf{H}_{i}$ is at least six, see [7], [20]. This is a $(\rho, \lambda)$ QC LDPC code based on Type-II EG.

\section{Self-orthogonal QC LDPC Codes}

We can define a self-orthogonal parity check matrix $\mathbf{H}_{E G, \ell}^{\text {orth }}$ from Type-II EG construction as follows. The binary matrix $\mathbf{H}_{E G, \ell}^{T}$ of size $n \times \ell n$ for $1 \leq \ell \leq\left(q^{m-1}-1\right) /(q-1)$ has row and column weights of $\lambda=\ell q$ and $\rho=q$, respectively. Let 1 be the column vector of size $\left(q^{m}-1\right) \times 1$ defined as $\mathbf{1}=(1,1, \ldots, 1)^{T}$. If the weight of a row in $\mathbf{H}_{E G, \ell}^{T}$ is odd, then we can add the vector $\mathbf{1}$ to form the matrix $\mathbf{H}_{E G, \ell}^{\text {orth }}=$ $\left[\mathbf{H}_{E G, \ell}^{T} \mid \mathbf{1}\right]$. Also, if the weight of a row in $\mathbf{H}_{E G, \ell}^{T}$ is even, then we can add the vector $\mathbf{1}$ along with the identity matrix of size $\left(q^{m}-1\right) \times\left(q^{m}-1\right)$ to form $\mathbf{H}_{E G, \ell}^{\text {orth }}=\left[\mathbf{H}_{E G, \ell}^{T}|\mathbf{1}|\right.$ $\mathbf{I}$. Therefore, we can prove that $\mathbf{H}_{E G, \ell}^{\text {orth }}$ is self-orthogonal as shown in the following Lemma.

Lemma 4: The parity check matrix $\mathbf{H}_{E G, \ell}^{\text {orth }}$ defined as

$$
\mathbf{H}_{E G, \ell}^{\text {orth }}=\left\{\begin{array}{l}
{\left[\begin{array}{llll}
\mathbf{H}_{1}^{T} & \mathbf{H}_{2}^{T} & \ldots & \mathbf{H}_{\ell}^{T} \mid \mathbf{1}
\end{array}\right], \text { for odd } \ell q ;} \\
{\left[\begin{array}{llll}
\mathbf{H}_{1}^{T} & \mathbf{H}_{2}^{T} & \ldots & \mathbf{H}_{\ell}^{T}|\mathbf{1}| \mathbf{I}
\end{array}\right], \text { for even } \ell q}
\end{array}\right.
$$

is self-orthogonal.

Proof: From the construction Type-II EG, any two different rows intersect (overlap) in exactly one nonzero position. If $\ell q$ is odd, then adding the column vector $\mathbf{1}$ will result an even overlap as well as rows of even weights. Therefore, the inner product mod 2 of any arbitrary rows vanishes. Also, if $\ell q$ is even, adding the columns $[\mathbf{1} \mid \mathbf{I}]$ will produce row of even weights and the inner product $\bmod 2$ of any arbitrary rows vanishes.

$\mathbf{H}_{E G, \ell}^{\text {orth }}$ has size $n \times N$ for odd $\ell q$ where $n=q^{m}-1$, $N=n \ell+1$, and $1 \leq \ell \leq\left(q^{(m-1)}-1\right) /(q-1)$. Also, it has length $N=n(\ell+1)+1$ for even $\ell$.

The minimum distance of the LDPC codes constructed in this type can be shown using the $\mathrm{BCH}$ bound as stated in the following result.

Lemma 5: The minimum distance of an LDPC defined by the parity check matrix $\mathbf{H}_{E G, \ell}^{\text {orth }}$ is at least $q+1$.

\section{Quantum LDPC Block Codes}

In this section we derive a family of LDPC stabilizer codes derived from LDPC codes based on finite geometries. Let $P=$ $\{I, X, Z, Y=i X Z\}$ be a set of Pauli matrices defined as

$$
I=\left(\begin{array}{ll}
1 & 0 \\
0 & 1
\end{array}\right), X=\left(\begin{array}{cc}
0 & 1 \\
1 & 0
\end{array}\right), Z=\left(\begin{array}{cc}
1 & 0 \\
0 & -1
\end{array}\right)
$$


and the matrix $Y$ is the combination of the matrices $X$ bitflip and $Z$ phase-flip defined as $Y=i X Z=\left(\begin{array}{cc}0 & -i \\ i & 0\end{array}\right)$. Clearly,

$$
X^{2}=Z^{2}=Y^{2}=I .
$$

A well-known method to construct quantum codes is by using the stabilizer formalism, see for example [1], [5], [9], [17] and references therein. Assume we have a stabilizer group $S$ generated by a set $\left\{S_{1}, S_{2}, \ldots, S_{n-k}\right\}$ such that every two row operators commute with each other. The error operator $S_{j}$ is a tensor product of $n$ Pauli matrices.

$$
S_{j}=E_{1} \otimes E_{2} \otimes \ldots \otimes E_{n}, \quad E_{i} \in P .
$$

$S_{j}$ can be seen as a binary vector of length $2 n$ [5], [17]. A quantum code $Q$ is defined as +1 joint eigenstates of the stabilizer $S$. Therefore, a codeword state $|\psi\rangle$ belongs to the code $Q$ if and only if

$$
S_{j}|\psi\rangle=|\psi\rangle \text { for all } S_{j} \in S .
$$

CSS Construction: Let $\mathbf{G}$ and $\mathbf{H}$ be two binary matrices define the classical code $C$ and dual code $C^{\perp}$, respectively. The CSS construction assumes that the stabilizer subgroup (matrix) can be written as

$$
\mathbf{S}=\left(\begin{array}{c|c}
\mathbf{H} & \mathbf{0} \\
\mathbf{0} & \mathbf{G}
\end{array}\right)
$$

where $\mathbf{H}$ and $\mathbf{G}$ are $k \times n$ matrixes satisfying $\mathbf{H G}^{T}=\mathbf{0}$. The quantum code with stabilizer $\mathbf{S}$ is able to encode $n-2 k$ logical qubits into $n$ physical qubits. If $\mathbf{G}=\mathbf{H}$, then the selforthgonality or dual-containing condition becomes $\mathbf{H H}^{T}=\mathbf{0}$. If $C$ is a code that has a parity check matrix $\mathbf{H}$, then $C^{\perp} \subseteq C$.

Constructing Dual-containing LDPC Codes: Let us construct the stabilizer matrix

$$
S_{s t a b}=\left(\begin{array}{c|c}
H_{X} & 0 \\
0 & H_{Z}
\end{array}\right) .
$$

The matrix $\mathbf{H}_{E G, \ell}^{\text {orth }}$ is a binary self-orthogonal matrix as shown in Section III-C. We replace every nonzero element in $\mathbf{H}_{E G, \ell}^{\text {orth }}$ by the Pauli matrix $X$ to form the matrix $H_{X}$. Similarly, we replace every nonzero element in $\mathbf{H}_{E G, \ell}^{\text {orth }}$ by the Pauli matrix $Z$ to form the matrix $H_{Z}$. Therefore the matrix $S_{s t a b}$ is also self-orthogonal. We can assume that the matrix $H_{X}$ corrects the bit-flip errors, while the matrix $H_{Z}$ corrects the phase-flip errors, see [1], [17].

Lemma 6: A quantum LDPC code $Q$ with rate $(n-2 k) / n$ is a code whose stabilizer matrix $S_{\text {stab }}$ of size $2 k \times 2 n$ has a pair $(\rho, \lambda)$ where $\rho$ is the number of non-zero error operators in a column and $\lambda$ is the number of non-zero error operators in a row. Furthermore, $S_{s t a b}$ is constructed from a binary selforthogonal parity check matrix $\mathbf{H}_{E G, \ell}^{\text {orth }}$ of size $k \times n$.

Using Lemma 6 and LDPC codes given by the parity check matrix $\mathbf{H}_{E G, \ell}^{\text {orth }}$ as shown in Section III-C, we can derive a class of quantum LDPC codes as stated in the following Lemma.

Theorem 7: Let $\mathbf{H}_{E G, \ell}^{\text {orth }}$ be a parity check matrix of an LDPC code based on $E G(m, q)$, where $n=q^{m}-1$ and
$1 \leq \ell \leq\left(q^{m-1}-1\right) /(q-1)$. Then, there exists a quantum LDPC code $Q$ with parameters $[[N, N-2 n, \geq q+1]]_{2}$ where $N=\ell n+1$ for odd $\ell q$ and $N=(\ell+1) n+1$ for even $\ell q$.

Proof: By Lemma 4, $\mathbf{H}_{E G, \ell}^{\text {orth }}$ is self-orthogonal. Using Lemma 6, there exists a quantum LDPC code with the given parameters.

\section{CONClusion}

We constructed a class of quantum LDPC codes derived from finite geometries. The constructed codes have high rates and their minimum distances are bounded. They only have one cycle of length four between any two rows and many cycles of length of at least six. A new class of quantum LDPC codes based on projective geometries can be driven in a similar way.

\section{REFERENCES}

[1] S. A. Aly. Quantum Error Control Codes. PhD thesis, Texas A\&M University, January 2008.

[2] S. A. Aly, A. Klappenecker, and P. Sarvepalli. Subsystem codes. Proceedings of the 45th Allerton Conference on Communication, Control, and Computing, Urbana, IL, September 2006.

[3] S. A. Aly, A. Klappenecker, and P. K. Sarvepalli. On quantum and classical BCH codes. IEEE Trans. Inform. Theory, 53(3):1183-1188, 2007.

[4] D. Bacon. Operator quantum error correcting subsystems for selfcorrecting quantum memories. Phys. Rev. A, 73(012340), 2006.

[5] A. R. Calderbank, E. M. Rains, P. W. Shor, and N. J. A. Sloane. Quantum error correction via codes over GF(4). IEEE Trans. Inform. Theory, 44:1369-1387, 1998.

[6] T. Camara, H. Ollivier, and J. P. Tillich. Constructions and performance of classes of quantum LDPC codes. 2005. quant-ph/00000.

[7] M.C. Davey and D.J.C. MacKay. Low density parity check codes over GF(q). IEEE Commun. Lett., 2(6):165-67, 1998.

[8] R.G. Gallager. Low density parity check codes. IRE Trans. Inform. Theory, 8, 1962.

[9] D. Gottesman. Stabilizer codes and quantum error correction. Caltech Ph. D. Thesis, eprint: quant-ph/9705052, 1997.

[10] M. Hagiwara and H. Imai. Quantum quasi-cyclic LDPC codes. submitted to ISIT07, 2007. quant-ph 701020v1.

[11] A. Ketkar, A. Klappenecker, S. Kumar, and P. K. Sarvepalli. Nonbinary stabilizer codes over finite fields. IEEE Trans. Inform. Theory, 52(11):4892-4914, 2006.

[12] Y. Kou, S. Lin, and M.P.C. Fossorier. Low-density parity-check codes based on finite geometries: A rediscovery and new results. IEEE Trans. Inform. Theory, 47(7):2711-2736, 2001.

[13] D. W. Kribs, R. Laflamme, D. Poulin, and M. Lesosky. Operator quantum error correction. Eprint: quant-ph/0504189, 2005.

[14] D.A. Lidar, I.L. Chuang, and K.B. Whaley. Decoherence-free subspaces for quantum-computation. Phys. Rev. Letters, 81:2594-2597, 1998.

[15] S. Lin and D.J. Costello. Error Control Coding. Pearson, Prentice Hall, 2004.

[16] G. Liva, S. Song, Y. Ryan W. Lan, L. Zhang, and S. Lin. Design of LDPC codes: A survey and new results. to appear in J. Comm. Software and Systems, 2006.

[17] D. J. C. MacKay, G. Mitchison, and P. L. McFadden. Sparse-graph codes for quantum error correction. IEEE Trans. Inform. Theory, 50(10):23152330, 2004.

[18] M.S. Postol. A proposed quantum low density parity check code. 2001. quant-ph/0108131.

[19] D. Poulin, J.P. Tillich, and H. Ollivier. Quantum serial turbo-codes. arXiv:0712.2888v1, 2007.

[20] S. Song, L. Zeng, S. Lin, and K. Abdel-Ghaffar. Algebraic constructions of nonbinary quasi-cyclic LDPC codes. Proc. 2006 IEEE Intl. Symp. Inform. Theory, pages 83-87, 2006.

[21] R.M. Tanner. A recursive approach to low complexity codes. IEEE Trans. Inform. Theory, 27:533-47, 1981. 